\title{
Fulde-Ferrell-Larkin-Ovchinnikov pairing in one-dimensional optical lattices
}

\author{
M. Rizzi, ${ }^{1,2}$ Marco Polini, ${ }^{2, *}$ M. A. Cazalilla, ${ }^{3,4}$ M. R. Bakhtiari, ${ }^{5}$ M. P. Tosi, ${ }^{2}$ and Rosario Fazio ${ }^{6,2}$ \\ ${ }^{1}$ Max-Planck-Institut für Quantenoptik, Hans-Kopfermann-Strasse 1, D-85748 Garching, Germany \\ ${ }^{2}$ NEST-CNR-INFM, I-56126 Pisa, Italy and Scuola Normale Superiore, I-56126 Pisa, Italy \\ ${ }^{3}$ Centro de Física de Materiales, Centro Mixto CSIC-UPV/EHU, P. Manuel de Lardizabal 3, 20018 San Sebastián, Spain \\ ${ }^{4}$ Donostia International Physics Center (DIPC), Manuel de Lardizabal 4, 20018 San Sebastian, Spain \\ ${ }^{5}$ Nanoscience Center, Department of Physics, University of Jyväskylä, P.O. Box 35, 40014 Jyväskylä, Finland \\ ${ }^{6}$ International School for Advanced Studies (SISSA), via Beirut 2-4, I-34014 Trieste, Italy
}

(Received 20 December 2007; revised manuscript received 17 March 2008; published 4 June 2008)

\begin{abstract}
Spin-polarized attractive Fermi gases in one-dimensional (1D) optical lattices are expected to be remarkably good candidates for the observation of the Fulde-Ferrell-Larkin-Ovchinnikov (FFLO) phase. We model these systems with an attractive Hubbard model with population imbalance. By means of the density-matrix renormalization-group method, we compute the pairing correlations as well as the static spin and charge structure factors in the whole range from weak to strong coupling. We demonstrate that pairing correlations exhibit quasi-long-range order and oscillations at the wave number expected from the FFLO theory. However, we also show by numerically computing the mixed spin-charge static structure factor that charge and spin degrees of freedom appear to be coupled already for a small imbalance. We discuss the consequences of this coupling for the observation of the FFLO phase, as well as for the stabilization of the quasi-long-range order into long-range order by coupling many identical 1D systems, such as in quasi-1D optical lattices.
\end{abstract}

DOI: 10.1103/PhysRevB.77.245105

PACS number(s): 71.10.Pm, 03.75.Ss, 03.75.Lm

\section{INTRODUCTION}

Multicomponent attractive fermionic systems with unequal masses, densities, or chemical potentials have attracted continued interest for many decades in several fields of physics ranging from high-energy ${ }^{1,2}$ to condensed matter ${ }^{2,3}$ and, more recently, atomic physics. ${ }^{4-6}$ The interplay between pairing and density imbalance of the different fermion species leads to a rich scenario, which includes the possibility of various exotic superconducting states. ${ }^{7}$ In this context, the Fulde-Ferrell-Larkin-Ovchinnikov (FFLO) phase $^{8}$ recently attracted a great deal of interest from both the experimental and the theoretical community. ${ }^{1-6}$ In the FFLO phase, Cooper pairing occurs between a fermion with momentum $\boldsymbol{k}$ and spin $\uparrow$ and a fermion with momentum $-\boldsymbol{k}+\boldsymbol{q}(\boldsymbol{q} \neq 0)$ and spin $\downarrow$. As a result, the superconducting order parameter becomes spatially dependent. Originally, the most favorable systems for the observation of the FFLO phase were predicted to be clean superconducting films in the presence of an in-plane (i.e., Zeeman) magnetic field, which is above the so-called Clogston-Chandrasekhar limit. ${ }^{9}$ Nevertheless, despite the fact that the original prediction dates back to more than 30 years ago, the FFLO phase has been very elusive to detect.

The experimental realization of interacting trapped Fermi gases with population imbalance ${ }^{4,5}$ renewed the hope of observing the FFLO, thus stimulating an intense theoretical activity. ${ }^{6,10}$ So far, most of the theoretical analysis has focused on three-dimensional (3D) cold atomic systems. However, as in the case of solid-state superconductors, the region of the phase diagram wherein the FFLO phase has been found to be stable is quite small. ${ }^{2,6}$ On the other hand, quasione-dimensional or strongly anisotropic systems (such as coupled chains and heavy-fermion, organic, high- $T_{c}$, and

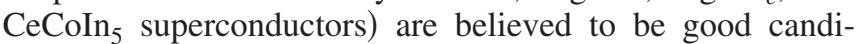

dates for the realization of the FFLO phase. ${ }^{2,3,11,12}$ Since the dimensionality of cold atomic systems can be easily tuned, and, indeed, cold atoms have already been successfully trapped in one-dimensional (1D) geometries, ${ }^{13}$ it seems natural to consider these low dimensional systems as the ideal candidates to observe nonhomogeneous pairing of the FFLO type.

Many important results are available on the properties of spin-polarized 1D Fermi systems with attractive interactions, which have been obtained by different methods and techniques. These include the Bethe-ansatz solutions of certain exactly solvable models such as the Hubbard or Gaudin-Yang models, ${ }^{14-17}$ as well as different types of numerical approaches such as density-matrix renormalization-group ${ }^{18-20}$ (DMRG) and quantum Monte Carlo $^{21}$ (QMC) or field theoretical techniques such as bosonization. ${ }^{12,18}$ Very recently Orso ${ }^{14}$ and Hu et al. ${ }^{15}$ have studied the phase diagram of harmonically trapped 1D polarized Fermi gases by combining the exact solution of the Gaudin-Yang model with a local-density approximation. Mean-field theory was also applied by Liu et al.,22 although it is known that it has a number of limitations ${ }^{23}$ in one dimension, particularly as far as paring correlations are concerned. DMRG was employed by Feiguin and Heidrich-Meisner ${ }^{19}$ and Tezuka and Ueda, ${ }^{20}$ and QMC was employed by Batrouni et $a .^{21}$ to investigate the pairing correlations in the spin-polarized ground state of the attractive Hubbard model in the presence of harmonic trapping. Previously, Yang ${ }^{12}$ used bosonization to study the pairing correlations and the phase diagram of a single 1D Fermi system as well as an array of weakly coupled 1D Fermi systems in the presence of a Zeeman field.

Yang's ${ }^{12}$ analysis is valid only close to a continuous magnetic-field-driven transition from a uniform BCS phase and an FFLO phase, which he assumed to belong to the commensurate-incommensurate universality class. ${ }^{24}$ Another 
important assumption in Ref. 12 is that charge and spin degrees of freedom are decoupled at low energies for a small polarization. However, this scenario does not apply to the Hubbard model away from half-filling ${ }^{17,25,26}$ nor to the Gaudin-Yang model, ${ }^{16}$ which are the relevant models for current $1 \mathrm{D}$ cold atomic systems. As we will show in this work, charge and spin degrees of freedom are indeed coupled already for a small polarization, which leads to important differences as compared to the scenario described by Yang. ${ }^{12}$ We also numerically demonstrate that the pairing correlation function exhibits prominent oscillations with a wave number equal (up to finite-size corrections, see below) to the difference of Fermi wave numbers, $q_{\mathrm{FFLO}}=\left|k_{F \uparrow}-k_{F \downarrow}\right|$, as predicted by the FFLO theory in one dimension ${ }^{12}$ and in agreement with a number of Luttinger-type theorems. ${ }^{27,28}$ Thus, the finite-wave-number oscillations in the pairing correlation function can be regarded as due to the excess of $n_{\uparrow}-n_{\downarrow}$ unpaired majority-spin fermions. ${ }^{12}$ This is because in one dimension, the Fermi wave number and the density are proportional to each other: $k_{F \sigma}=\pi n_{\sigma}$. On the other hand, this relationship is no longer linear in dimensionality higher than one, and in this case, the oscillations in the order parameter are related to the center-of-mass momentum $\boldsymbol{q}$ of the Cooper pairs. These observations seem to indicate that, in the 1D case, it is not entirely clear whether there is a strict close parallelism with higher dimensional FFLO, and in some respects, the system can be also understood as a coupled BoseFermi mixture of spin-singlet pairs (the bosons) and unpaired fermions. ${ }^{15,26}$

The paper is organized as follows. Section II presents the Hamiltonian that we use to describe the system of physical interest, while Sec. III reports and discusses our main numerical results. Our main conclusions are briefly reported in Sec. IV.

\section{MODEL}

We consider a two-component mixture with a total of $N$ fermionic atoms loaded in a 1D optical lattice with $L$ sites (the lattice constant is taken to be unity). An explicit example is provided by mixtures of two hyperfine states of ${ }^{6} \mathrm{Li}$, as in the pioneering experiments of Ref. 4. The fermions are assumed to interact via attractive on-site interactions, whose strength can be tuned, e.g., by means of a Feshbach resonance. Sufficiently away from resonance(s), this system is modeled by the attractive Hubbard model, as proven in Ref. 29:

$$
\hat{\mathcal{H}}=-t \sum_{\sigma, \ell=1}^{L-1}\left(\hat{c}_{\ell \sigma}^{\dagger} \hat{c}_{\ell+1 \sigma}+\text { H.c. }\right)-U \sum_{\ell=1}^{L} \hat{n}_{\ell \uparrow} \hat{n}_{\ell \downarrow},
$$

where $t$ is the hopping parameter, $\hat{c}_{\ell \sigma}^{\dagger}\left(\hat{c}_{\ell \sigma}\right)$ is the creation (destruction) fermion operator in the $\ell$ site $(\ell \in[1, L]), \sigma$ $=\uparrow, \downarrow$ is the pseudospin- $1 / 2$ index (in experiments, this labels the two different atomic hyperfine states of the mixture), $U>0$ is the strength of the on-site Hubbard attraction, $\hat{n}_{\ell \sigma}$ $=\hat{c}_{\ell \sigma}^{\dagger} \hat{c}_{\ell \sigma}$, and $N_{\sigma}=\Sigma_{\sigma} \hat{n}_{\ell \sigma}$. External parabolic trapping potentials may give rise to spurious effects such as phase separation. ${ }^{15}$ In order to disentangle such behaviors from fundamental effects related to nonconventional FFLO-type pairing, we have deliberately decided to limit our present investigations to a homogeneous external potential. In this context, simple open boundary conditions (OBCs) are the natural type of boundary conditions for DMRG, as originally formulated by White. ${ }^{30}$ Moreover, such conditions may turn out to be useful for recent atom chip experiments. ${ }^{31}$ Our calculations are performed in the canonical ensemble, and the results apply only to lattices away from half-filling, that is, when $N \neq L$. In the calculations, the spin polarization $\delta$ $=\left(N_{\uparrow}-N_{\downarrow}\right) /\left(N_{\uparrow}+N_{\downarrow}\right)$ was varied by decreasing $N_{\downarrow}$ while keeping constant the number of "background" up-spin atoms $N_{\uparrow}$ from $N_{\downarrow}=N_{\uparrow}$ (the unpolarized case, i.e., $\delta=0$ ) all the way down to $N_{\downarrow}=0$ (the fully polarized case, i.e., $\delta=1$ ).

In the unpolarized case $(\delta=0)$, all fermions pair into spin singlets due to the attractive on-site interaction. This yields a gap to all spin excitations, and therefore, spin-spin correlations exponentially decay with distance. Singlet pairing and charge-density wave correlations exhibit a slower decay (of power-law type in the ground state of a thermodynamically large system), with singlet pairing correlations the ones that dominate at long distances in systems away from half-filling. ${ }^{32}$ The aim of this work is to study the nature of pairing for $0<\delta<1$ as a function of the dimensionless ratio $U / t$ (in the fully polarized case, where $\delta=1, \hat{\mathcal{H}}$ describes a system of $N=N_{\uparrow}$ noninteracting fermions). The expectation values $\langle\cdots\rangle$ of all operators below are understood to be taken over the ground state of $\hat{\mathcal{H}}$.

\section{NUMERICAL RESULTS AND DISCUSSION}

Due to the OBCs (or, in general, to any external potential that breaks the Bloch translational invariance of the lattice), the spin-resolved site occupation profiles, $n_{\ell \sigma}=\left\langle\hat{n}_{\ell \sigma}\right\rangle$, exhibit Friedel oscillations. This is illustrated in Fig. 1. In the unpolarized $\delta=0$ case, the Friedel oscillations in $n_{\uparrow}$ are in phase with those in $n_{1}$, giving rise to large-amplitude atomicdensity waves ${ }^{33,34}$ in the total site occupation $n_{\ell \uparrow}+n_{\ell \downarrow}$. As it is clear from the top panel of Fig. 1, in the general $\delta \neq 0$ case, the total site occupation displays $N_{\downarrow}$ maxima associated with the formation of $N_{\downarrow}$ spin-singlet pairs that are delocalized over the lattice. In the bottom panel of Fig. 1, we show the local spin polarization $n_{\ell \uparrow}-n_{\ell \downarrow}$ (which could be measured through phase-sensitive optical imaging ${ }^{5}$ ). For small $\delta$ (see, e.g., the plot for $N_{\uparrow}=20$ and $N_{\downarrow}=16$ ), the local spin polarization displays $N_{\uparrow}-N_{\downarrow}$ maxima corresponding to the number of fermions that are left unpaired. With increasing $\delta$, although the spatial dependence of the local spin polarization becomes more complicated, the amplitude of the oscillations in the bulk becomes indeed smaller, thus making it hard to clearly identify $N_{\uparrow}-N_{\downarrow}$ maxima. These, however, are not distinctive and unambiguous signals of the FFLO pairing.

We thus proceed below to present a study of pairing correlations: the model described in Eq. (1), in fact, cannot sustain any true long-range order ${ }^{32}$ in one dimension, i.e., the ground-state expectation value of the pairing operator $\hat{\Delta}_{\ell}$ $=\hat{c}_{\ell \downarrow} \hat{c}_{\ell \uparrow}$ is zero.

In the unpolarized case and for an extended system, the correlation function of the pairing operator $\mathcal{C}_{\ell \ell^{\prime}}=\left\langle\hat{\Delta}_{\ell}^{\dagger} \hat{\Delta}_{\ell^{\prime}}\right\rangle$ de- 

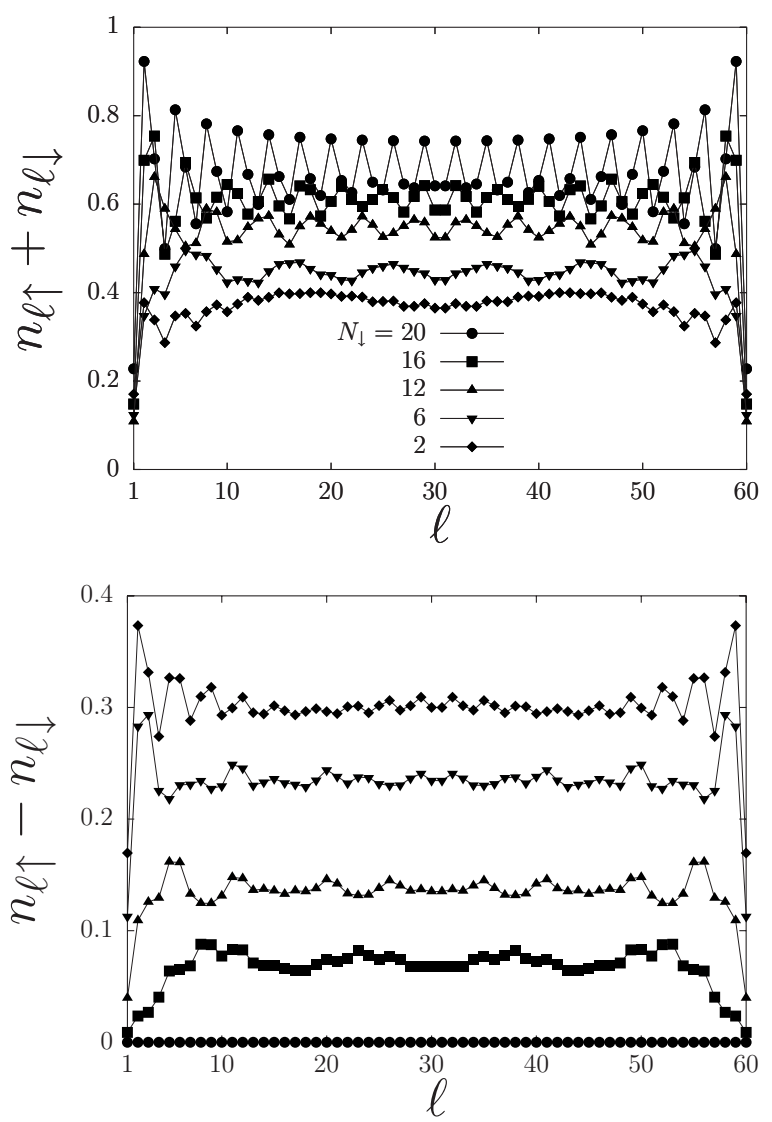

FIG. 1. Top panel: DMRG results for the total site occupation $n_{\ell \uparrow}+n_{\ell \downarrow}$ as a function of site position $\ell$ for a system with $N_{\uparrow}=20$ fermions in $L=60$ lattice sites and $U / t=5$. The numbers of downspin fermions are $N_{\downarrow}=20,16,12,6$, and 2 (the corresponding spin polarization is $\delta=0 \%, 11 \%, 25 \%, 54 \%$, and $82 \%$ ). Bottom panel: the local spin polarization $n_{\ell \uparrow}-n_{\ell \downarrow}$ as a function of $\ell$ for the same system parameters as in the top panel. The thin solid lines are just guides to the eye.

cays with a power law $\left|\ell-\ell^{\prime}\right|^{-1 / K_{\rho}}$ at large distances, where $1 \leqslant K_{\rho} \leqslant 2$ is an interaction-dependent Luttinger-liquid dimensionless parameter. ${ }^{32}$ In the top panel of Fig. 2, we illustrate our DMRG results for the spin-polarization dependence of $\mathcal{C}_{\ell \ell^{\prime}=L / 2}$ at $U / t=5$, which measures real-space pairing correlations between the site $\ell^{\prime}=L / 2$ (the center of the trap) and all the other sites. For $\delta=0$, the power-law decay of the $\mathcal{C}_{\ell \ell^{\prime}=L / 2}$ for $|\ell-L / 2| \gg 1$ is clearly visible. For finite $\delta$, instead, the pairing correlator is characterized by a distinctive oscillatory character ${ }^{12}$ and a very simple nodal structure with exactly $N_{\uparrow}-N_{\downarrow}$ zeros. We have carefully checked that the long-distance decay of $\mathcal{C}_{\ell \ell^{\prime}=L / 2}$ is still power law, signaling a quasi-long-range pairing behavior also at finite $\delta$.

A careful analysis of the oscillatory character of $\mathcal{C}_{\ell \ell^{\prime}=L / 2}$ can be done by means of the Fourier transform of the pairing correlator,

$$
\mathcal{C}\left(k_{m}, k_{m^{\prime}}\right)=\mathcal{F} \mathcal{T}\left[\mathcal{C}_{\ell \ell^{\prime}}\right]=\sum_{\ell, \ell^{\prime}=1}^{L} \mathcal{C}_{\ell \ell^{\prime}} \varphi_{m}(\ell) \varphi_{m^{\prime}}\left(\ell^{\prime}\right) .
$$

where $\varphi_{m}(\ell)=[2 /(L+1)]^{-1 / 2} \sin \left(k_{m} \ell\right)\left[\right.$ with $k_{m}=\pi m /(L+1)$, $m=1, \ldots, L]$ are the eigenstates of the hopping term in Eq.
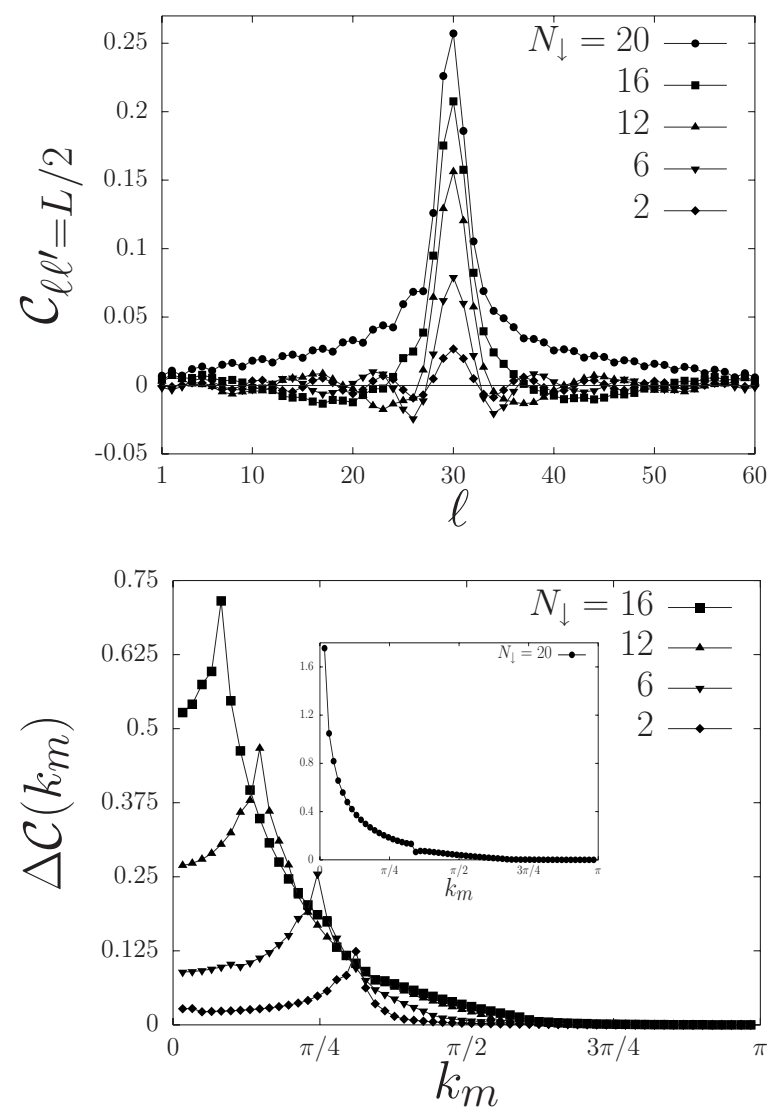

FIG. 2. Top panel: DMRG results for the pairing correlator $\mathcal{C}_{\ell \ell^{\prime}=L / 2}$ as a function of site position $\ell$ for a system with $N_{\uparrow}=20$ fermions in $L=60$ lattice sites and $U / t=5$. The numbers of downspin fermions are $N_{\downarrow}=20,16,12,6$, and 2 (the corresponding spin polarization is $\delta=0 \%, 11 \%, 25 \%, 54 \%$, and $82 \%$ ). Bottom panel: Interaction contribution to the momentum-space pairing correlator $\Delta \mathcal{C}\left(k_{m}\right)$ as a function of wave number $k_{m}=\pi m /(L+1)$. The symbol coding is the same as in the top panel. Note the well-defined peaks at $q_{\text {FFLO }}$ In the inset, we show $\Delta \mathcal{C}\left(k_{m}\right)$ for the unpolarized system with $N_{\uparrow}=N_{\downarrow}=20$, which shows a narrow peak at $k_{1}$. The thin solid lines are just guides to the eye.

(1). The mode with zero wave number is excluded from the allowed $k_{m}$ values due to the OBC. The lowest energy mode corresponds to $k_{1}$. The diagonal part of the matrix $\mathcal{C}\left(k_{m}, k_{m^{\prime}}\right)$ will be simply denoted by $\mathcal{C}\left(k_{m}\right) \equiv \operatorname{diag}\left\{\mathcal{C}\left(k_{m}, k_{m^{\prime}}\right)\right\}$ $=\mathcal{C}\left(k_{m}, k_{m}\right)$. In the bottom panel of Fig. 2, we plot the difference $\Delta \mathcal{C}\left(k_{m}\right)=\mathcal{C}\left(k_{m}\right)-\mathcal{C}^{(0)}\left(k_{m}\right)$ between $\mathcal{C}\left(k_{m}\right)$ and its value in the noninteracting gas (i.e., at $U / t=0), \mathcal{C}^{(0)}\left(k_{m}\right) .{ }^{35}$

At $\delta=0, \mathcal{C}\left(k_{m}\right)$ possesses a very narrow peak at $k_{1}$ (see inset in the bottom panel of Fig. 2), signaling quasi-longrange pairing order of the conventional BCS type. For a finite $\delta$, instead, $\mathcal{C}\left(k_{m}\right)$ has a local minimum at $k_{1}$ and a single well-defined peak appears at a wave number $q_{\text {FFLO }}=k_{1}$ $+\left|k_{F \uparrow}-k_{F \downarrow}\right|$, where $k_{F \sigma}=\pi N_{\sigma} /(L+1)$ are the spin-resolved Fermi wave numbers. The peak at $q_{\text {FFLO }}$ in the Fourier transform of the pairing correlator, which is a direct consequence of the simple real-space nodal structure illustrated in the top panel of Fig. 2, is a clear-cut signal of FFLO pairing. The numerical results shown in Fig. 2 are in perfect agreement with similar results obtained by means of the QMC method which have appeared recently in the literature. ${ }^{21} \mathrm{We}$ instead 

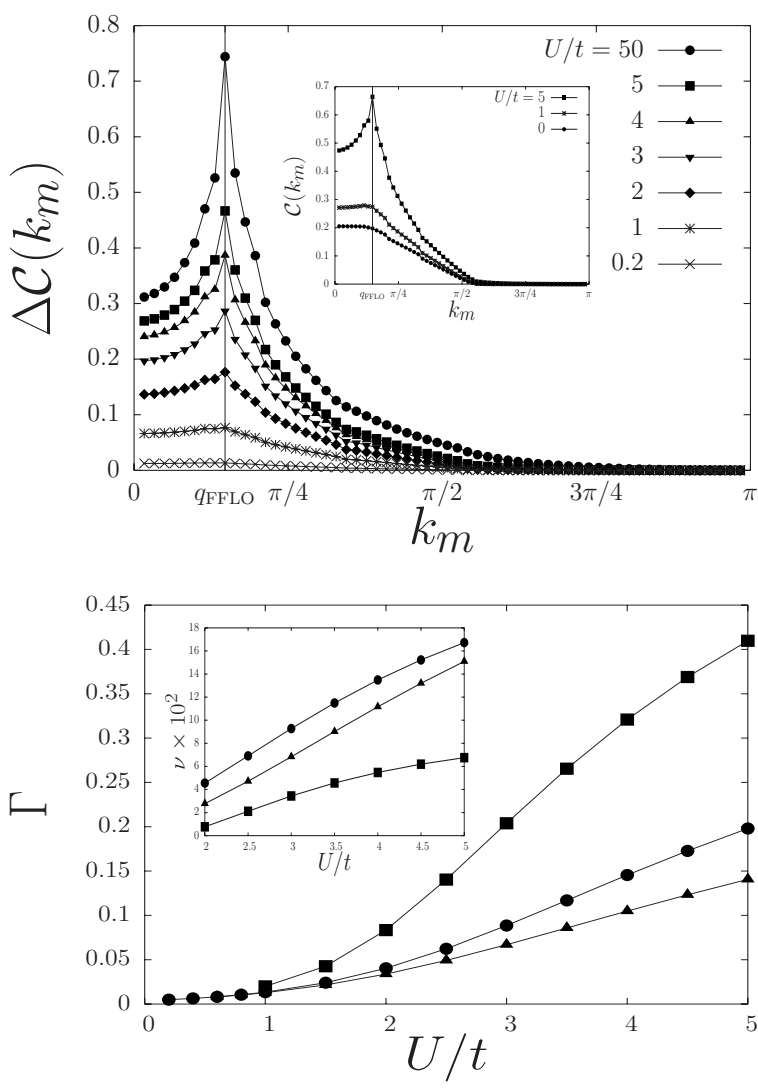

FIG. 3. Top panel: Interaction contribution to the momentumspace pairing correlator $\Delta \mathcal{C}\left(k_{m}\right)$ as a function of $k_{m}$ for $N_{\uparrow}=20$, $N_{\downarrow}=12(\delta=25 \%)$, and $L=60$. The interaction strength $U / t$ is decreased from 50 to 0.2 . In the inset, we show $\mathcal{C}\left(k_{m}\right)$ for $U / t=5,1$, and 0 . Bottom panel: The FFLO anomaly $\Gamma$ in the pair correlator [see Eq. (3)] as a function of $U / t$ for $N_{\uparrow}=20$ and $N_{\downarrow}=18$ (squares, $\delta=5 \%$ ), $N_{\downarrow}=12$ (circles, $\delta=25 \%$ ), and $N_{\downarrow}=6$ (triangles, $\delta=54 \%$ ). Inset: peak visibility $\nu$ [see Eq. (4)] as a function of $2 \leqslant U / t \leqslant 5$ for the same system parameters as in the main body of the figure ( $\nu$ is about $30 \%$ at $U / t=50$ ). The thin solid lines are just guides to the eye.

refer the reader to Refs. 19 and 20 for numerical studies of the impact of a harmonic confinement on the pairing correlations.

The DMRG data shown in Fig. 2 refer only to a single value of $U / t=5$. We now turn to illustrate the dependence of the pairing correlation functions on $U / t$. In the top panel of Fig. 3, we illustrate the dependence of $\Delta \mathcal{C}\left(k_{m}\right)$ on the interaction strength $U / t$ for a fixed spin polarization $\delta=25 \%$. On decreasing $U / t$, the quasi-long-range FFLO order (i.e., the height of the peak at $\left.q_{\mathrm{FFLO}}\right)$, which is emphatically strong for large $U / t$, survives all the way down to the weak coupling regime. This can be quantified better by analyzing the size of the anomaly $\Gamma$ at $k_{m}=q_{\text {FFLO }}$, which is measured by the difference between the left and right (discrete) derivatives of $\mathcal{C}\left(k_{m}\right)$ evaluated at $q_{\text {FFLO }}$,

$$
\Gamma=\mathcal{C}\left(q_{\mathrm{FFLO}}+k_{1}\right)+\mathcal{C}\left(q_{\mathrm{FFLO}}-k_{1}\right)-2 \mathcal{C}\left(q_{\mathrm{FFLO}}\right) .
$$

In the bottom panel of Fig. 3, we plot $\Gamma$ as a function of $U / t \leqslant 5$. In this range, $\Gamma$ decreases in a smooth fashion to its noninteracting value (i.e., $\Gamma=0)$ as $U / t$, is decreased to zero. In other words, for every finite $\delta, \mathcal{C}\left(k_{m}\right)$ uniformly and smoothly tends to its noninteracting value $\mathcal{C}^{(0)}\left(k_{m}\right)$ as $U / t$ is decreased toward zero. For sufficiently large values of $U / t$, the FFLO phase can be also characterized by the peak visibility defined by

$$
\nu=\frac{\mathcal{C}\left(q_{\mathrm{FFLO}}\right)-\mathcal{C}\left(k_{1}\right)}{\mathcal{C}\left(q_{\mathrm{FFLO}}\right)+\mathcal{C}\left(k_{1}\right)} .
$$

This quantity is plotted in an inset in the bottom panel of Fig. 3.

Before concluding, we would like to illustrate the behavior of the density-density, spin-spin, and mixed density-spin static structure factors, $S_{n n}\left(k_{m}\right), S_{m m}\left(k_{m}\right)$, and $S_{n m}\left(k_{m}\right)$. These are defined by the sum over all frequencies of the corresponding dynamic structure factors ${ }^{36}$ that can be, in principle, measured through Bragg spectroscopy or Fourier sampling of time-of-flight images. ${ }^{37}$ In practice, $S_{n n}\left(k_{m}\right)$, $S_{m m}\left(k_{m}\right)$, and $S_{n m}\left(k_{m}\right)$ are calculated from the following equations:

$$
\begin{gathered}
S_{n n}\left(k_{m}\right)=\operatorname{diag}\left\{\mathcal{F} \mathcal{T}\left[\left\langle\hat{n}_{\ell} \hat{n}_{\ell^{\prime}}\right\rangle-\left\langle\hat{n}_{\ell}\right\rangle\left\langle\hat{n}_{\ell^{\prime}}\right\rangle\right]\right\}, \\
S_{m m}\left(k_{m}\right)=\operatorname{diag}\left\{\mathcal{F} \mathcal{T}\left[\left\langle\hat{m}_{\ell} \hat{m}_{\ell^{\prime}}\right\rangle-\left\langle\hat{m}_{\ell}\right\rangle\left\langle\hat{m}_{\ell^{\prime}}\right\rangle\right]\right\}, \\
S_{n m}\left(k_{m}\right)=\operatorname{diag}\left\{\mathcal{F} \mathcal{T}\left[\left\langle\hat{n}_{\ell} \hat{m}_{\ell^{\prime}}\right\rangle-\left\langle\hat{n}_{\ell}\right\rangle\left\langle\hat{m}_{\ell^{\prime}}\right\rangle\right]\right\},
\end{gathered}
$$

where $\hat{n}_{\ell}=\hat{n}_{\ell \uparrow}+\hat{n}_{\ell \downarrow}$ and $\hat{m}_{\ell}=\hat{n}_{\ell \uparrow}-\hat{n}_{\ell \downarrow}$. In Figs. 4 and 5, we show the dependence of $S_{n n}\left(k_{m}\right), S_{m m}\left(k_{m}\right)$, and $S_{n m}\left(k_{m}\right)$ on $U / t$ for a slightly asymmetric system with $N_{\uparrow}=20$ and $N_{\downarrow}$ $=18(\delta \sim 5 \%)$. We remind the reader that in the unpolarized $\delta=0$ case, $S_{n n}\left(k_{m}\right)$ has a peak at $k_{m}=2 k_{F \uparrow}=2 k_{F \downarrow}$ that signals real-space atomic-density waves. ${ }^{33,34}$ In the spin-polarized case, this peak splits into two peaks at $2 k_{F \uparrow}$ and $2 k_{F \downarrow}$. This is clearly visible in Fig. 4 in the static density-density structure factor (top panel), which presents a double-peak structure slightly below $k_{m}=3 \pi / 4\left(2 k_{F \uparrow} \approx 2 \pi / 3\right.$ and $2 k_{F \downarrow} \approx 3 \pi / 5$ for the system parameters in this figure). This double-peak structure is not so visible in the magnetic structure factor $S_{m m}\left(k_{m}\right)$, most likely because magnetic correlations near $2 k_{F \uparrow}$ and $2 k_{F \downarrow}$ are still quite suppressed by the pairing correlations, at least in the weakly polarized case (in the unpolarized case, they are completely suppressed by the pairing gap). From Fig. 5, we note that $S_{n m}\left(k_{m}\right)$ is nonzero even at small $k_{m}$, thus indicating that spin and charge degrees of freedom are coupled at long wavelengths even for a small imbalance.

\section{A. Experimental signatures of the Fulde-Ferrell-Larkin- Ovchinnikov phase}

The most direct way to detect FFLO pairing would be to measure the pairing correlation function $\mathcal{C}_{\ell \ell^{\prime}}$. We would like to remark here that this correlation function is, at least in principle, measurable via interferometric schemes ${ }^{38}$ in which two atomic wave packets are coherently extracted from the gas at different positions and then are mixed by a matterwave beam splitter. The atom counting statistics in the beam splitter output channels has been shown ${ }^{38}$ to reflect the spatial dependence of $\mathcal{C}_{\ell \ell^{\prime}}$. 

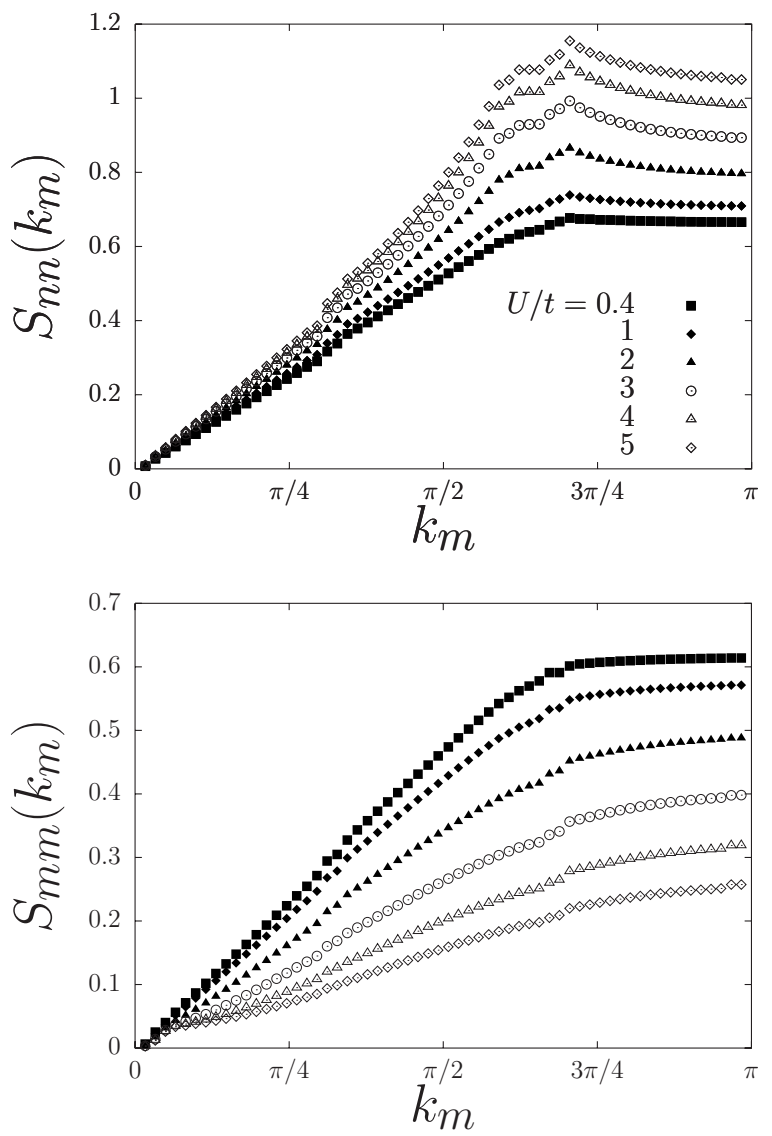

FIG. 4. Top panel: the density-density structure factor $S_{n n}\left(k_{m}\right)$ as a function of $k_{m}$ for $N_{\uparrow}=20$ and $N_{\downarrow}=18$ and different values of $U / t=0.4,1,2,3,4$, and 5 (from bottom to top). Bottom panel: the spin-spin structure factor $S_{m m}\left(k_{m}\right)$. The symbol coding is as in the top panel.

The oscillations of the pairing correlations will also leave a detectable signature in the noise correlations, ${ }^{39,40}$ $G_{\uparrow \downarrow}\left(\boldsymbol{k}, \boldsymbol{k}^{\prime}\right)=\left\langle\hat{n}_{\boldsymbol{k}, \uparrow} \hat{n}_{\boldsymbol{k}^{\prime}, \downarrow}\right\rangle-\left\langle\hat{n}_{\boldsymbol{k}, \uparrow}\right\rangle\left\langle\hat{n}_{\boldsymbol{k}^{\prime}, \downarrow}\right\rangle$, where $\hat{n}_{\boldsymbol{k}, \sigma}$ measures the number of fermions with momentum $\boldsymbol{k}$ and spin $\sigma$ in a timeof-flight experiment. With increasing the spin polarization, in fact, the peak at $\boldsymbol{k}=-\boldsymbol{k}^{\prime}=(k, 0,0)$ (Refs. 41 and 42) [here $(1,0,0)$ is the direction along the axis of the $1 \mathrm{D}$ system] will shift to a finite relative momentum (see, e.g., the work by Yang in Ref. 10 and the very recent DMRG calculation by Lüscher et $a l .{ }^{43}$ ).

However, it is worth pointing out that the strength of the noise signal in a strictly $1 \mathrm{D}$ system will be strongly affected by finite-size and temperature effects. This is because in one dimension, the order is not long range but quasi-long-range, and therefore, the slowest decay exhibited by correlations (such as the pairing correlations) is at most a power law. Thus, in order to enhance the strength of the experimental signal for FFLO, it would be desirable to couple many 1D systems, as in a tight two-dimensional optical lattice (arrays of "atomic quantum wires"), ${ }^{44}$ so that the quasi-long-range FFLO order can become a true long-range order. The phase diagram of many coupled 1D systems has been worked out in Ref. 12, where the author found that, at small polarization $\delta$, true long-range 3D FFLO order will occur when the Luttinger-liquid parameter for the charge excitations, $K_{\rho}$, is

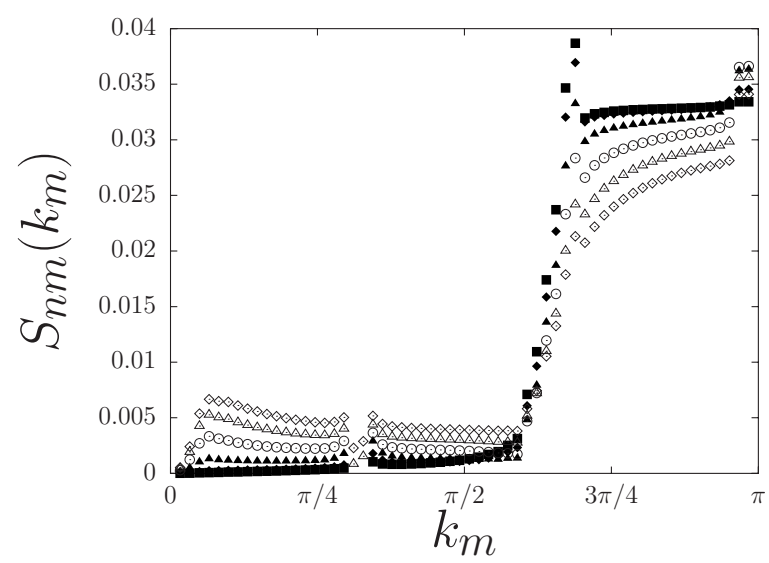

FIG. 5. The mixed density-spin structure factor $S_{n m}\left(k_{m}\right)$ as a function of $k_{m}$ for $N_{\uparrow}=20$ and $N_{\downarrow}=18$ and different values of $U / t$ $=0.4,1,2,3,4$, and 5. The symbol coding is as in Fig. 4. Whereas the results at small $U / t$ (see, for example, filled squares corresponding to $U / t=0.4$ ) are likely to be somewhat affected by finite-size effects, which prevent the full development of a pairing gap, the results at larger $U$ clearly show a nonvanishing weight of $S_{n m}\left(k_{m}\right)$ at small $k_{m}$, thus indicating that spin and charge degrees of freedom are coupled at long wavelengths.

larger than $3 / 2$. In such a case, the low temperature properties of the system are dominated by hopping of pairs (i.e., Josephson coupling) rather than by single-particle hopping (the latter would turn the system into an anisotropic Fermi liquid, which could in turn become unstable to the FFLO state under appropriate conditions ${ }^{45}$ ).

However, the analysis of Ref. 12 assumed the transition from the unpolarized to the polarized case to belong to the commensurate-incommensurate universality class. This assumption implicitly neglects the coupling between charge and spin degrees of freedom at low energies, which is known to modify the behavior of physical observables at the transition. ${ }^{25,26}$ In this work, this coupling has been demonstrated to also exist at long wavelengths by an explicit numerical evaluation of the mixed static structure factor $S_{n m}\left(k_{m}\right)$ in a weakly polarized system (see Fig. 5). Thus, the phase diagram depicted in Fig. 1 of Ref. 12 seems not appropriate for coupled 1D Hubbard (or Gaudin-Yang ${ }^{16}$ ) models, and the transition to long-range order will not take place in general for $K_{\rho}=3 / 2$ and may in general depend on the system parameters (i.e., $U / t$ and the lattice filling for the Hubbard model).

To the best of our knowledge, a quantitative phase diagram of coupled 1D systems lacking spin-charge separation has not yet been calculated. Furthermore, it is worth noticing that in cold atomic systems with short range interactions, another important factor must be taken into account, namely, the relative strength of the pair hopping when compared to the single-particle hopping. The strength of the latter is given by $t_{\perp}\left(t_{\perp} \ll \varepsilon_{F}\right.$, where $\varepsilon_{F}$ is the Fermi energy, for the analysis based on coupled Tomonaga-Luttinger liquids to hold), where $t_{\perp}$ is the hopping amplitude between two neighboring $1 \mathrm{D}$ systems. However, in the absence of long-range interactions, pair hopping can only be generated by (virtual) singleparticle tunneling events, which at lowest order, yield a (Jo- 
sephson) coupling strength of order $t_{\perp}^{2} / \Delta_{\sigma}$, where $\Delta_{\sigma}$ is the spin gap. The phase diagram predicted in Ref. 12 is the result of a calculation which only compares the scaling dimensions of the pair hopping and single-particle hopping operators, and thus does not take into account the microscopic details of the coupling between 1D systems. Thus, the stabilization of FFLO long-range order by a weak coupling between 1D systems in the FFLO phase (the extreme anisotropic limit that could not be accessed by the authors of Ref. 45) does not seem easily achievable. In turn, the most likely scenario for arbitrary polarization is that the single-particle hopping will control the physics at low temperatures, and the system will behave as a spin-polarized normal Fermi liquid, which, in turn, could become unstable toward 3D FFLO ordering ${ }^{45}$ under appropriate conditions.

In addition to the consequences for long-range FFLO order discussed above, the coupling between charge will also have other dramatic experimental consequences. Indeed, an experiment like the one described in Refs. 46-48 trying to reveal spin-change separation in a weakly polarized system, would fail to do so. In brief, we expect that creation of a pure total density (conversely, spin-density) fluctuation, using appropriate laser pulses, ${ }^{46,47}$ will also lead to the excitation of spin-density (total density) modes, thus revealing the spincharge coupling that we numerically find in the $S_{n m}(k)$ correlations (see Fig. 5). The coupling should also have importance consequences for the low frequency modes of harmonically trapped spin-polarized attractive Fermi gases, which will can be expected not to have a well-defined spin and charge character.

\section{CONCLUSIONS}

In summary, we have shown how ultracold spin-polarized two-component Fermi gases confined in 1D optical lattices are FFLO paired states whose pairing correlation functions are characterized by a power-law decay and a simple nodal structure. However, we have also shown that charge and spin degrees of freedom appear to be coupled already for a small value of the spin polarization. Finally, we have commented on the impact of this coupling on the detectability of true long-range FFLO order arising from Josephson coupling between $1 \mathrm{D}$ systems.

\section{ACKNOWLEDGMENTS}

We acknowledge useful discussions with P. Calabrese, I. Carusotto, M. Köhl, and A. H. MacDonald. M.P. gratefully acknowledges the hospitality of the Donostia International Physics Center and of the Department of Physics of the Zhejiang Normal University during the final stages of this work. M.A.C. thanks A. Nersesyan for drawing attention to Ref. 26. M.R. acknowledges the EU-project SCALA (015714). This work was partly supported by PRIN-MIUR and by the Academy of Finland (Project No. 115020). M. A. C. acknowledges financial support from MEC (Spain) under Grants No. FIS2004-06490-C03-00 and FIS2007-66711C02-02. The DMRG calculations have been made by using the DMRG code released within the "Powder with Power" Project (www.dmrg.it). *m.polini@sns.it

${ }^{1}$ K. Rajagopal and F. Wilczek, in Frontier of Particle Physics, edited by M. Shifman (World Scientific, Singapore, 2002).

${ }^{2}$ R. Casalbuoni and G. Nardulli, Rev. Mod. Phys. 76, 263 (2004).

${ }^{3} \mathrm{~K}$. Yang, in Pairing in Fermionic Systems: Basic Concepts and Modern Applications, edited by M. Alford, J. Clark, and A. Sedrakian (World Scientific, Singapore, 2006).

${ }^{4}$ M. W. Zwierlein, A. Schirotzek, C. H. Schunck, and W. Ketterle, Science 311, 492 (2006); G. B. Partridge, W. Li, R. I. Kamar, Y. Liao, and R. G. Hulet, ibid. 311, 503 (2006); M. W. Zwierlein, C. H. Schunck, A. Schirotzek, and W. Ketterle, Nature (London) 442, 54 (2006).

${ }^{5}$ Y. Shin, M. W. Zwierlein, C. H. Schunck, A. Schirotzek, and W. Ketterle, Phys. Rev. Lett. 97, 030401 (2006); G. B. Partridge, W. Li, Y. A. Liao, R. G. Hulet, M. Haque, and H. T. C. Stoof, ibid. 97, 190407 (2006).

${ }^{6}$ M. M. Parish, F. M. Marchetti, A. Lamacraft, and B. D. Simons, Nat. Phys. 3, 124 (2007).

${ }^{7}$ G. Sarma, J. Phys. Chem. Solids 24, 1029 (1963); H. Müther and A. Sedrakian, Phys. Rev. Lett. 88, 252503 (2002); W. V. Liu and F. Wilczek, ibid. 90, 047002 (2003); P. F. Bedaque, H. Caldas, and G. Rupak, Phys. Rev. Lett. 91, 247002 (2003).

${ }^{8}$ P. Fulde and R. A. Ferrell, Phys. Rev. 135, A550 (1964); A. J. Larkin and Y. N. Ovchinnikov, Zh. Eksp. Teor. Fiz. 47, 1136 (1964) [Sov. Phys. JETP 20, 762 (1965)].
${ }^{9}$ In a superconductor in the presence of Zeeman splitting, the Clogston-Chandrasekhar limit determines the critical field below which a uniform BCS order parameter can exist. Above this field, the electron system is fully polarized [B. S. Chandrasekhar, Appl. Phys. Lett. 1, 7 (1962); A. M. Clogston, Phys. Rev. Lett. 9, 266 (1962)].

${ }^{10}$ See, for example, R. Combescot, Europhys. Lett. 55, 150 (2001); T. Mizushima, K. Machida, and M. Ichioka, Phys. Rev. Lett. 94, 060404 (2005); K. Yang, ibid. 95, 218903 (2005); J. Carlson and S. Reddy, ibid. 95, 060401 (2005); D. E. Sheehy and L. Radzihovsky, ibid. 96, 060401 (2006); P. Pieri and G. C. Strinati, ibid. 96, 150404 (2006); H. Hu and X.-J. Liu, Phys. Rev. A 73, 051603(R) (2006); M. Haque and H. T. C. Stoof, ibid. 74, 011602 (2006); M. Mannarelli, G. Nardulli, and M. Ruggieri, ibid. 74, 033606 (2006); Y.-P. Shim, R. A. Duine, and A. H. MacDonald, ibid. 74, 053602 (2006); J. Kinnunen, L. M. Jensen, and P. Törmä, Phys. Rev. Lett. 96, 110403 (2006); T. N. De Silva and E. J. Mueller, ibid. 97, 070402 (2006); C.-C. Chien, Q. Chen, Y. He, and K. Levin, ibid. 97, 090402 (2006); K. Machida, T. Mizushima, and M. Ichioka, ibid. 97, 120407 (2006); C.-C. Chien, Q. Chen, Y. He, and K. Levin, ibid. 98, 110404 (2007) and references therein.

${ }^{11}$ T. Pruschke and H. Shiba, Phys. Rev. B 46, 356 (1992).

${ }^{12}$ K. Yang, Phys. Rev. B 63, 140511(R) (2001).

${ }^{13}$ See, e.g., B. Paredes, A. Widera, V. Murg, O. Mandel, S. Fölling, 
I. Cirac, G. V. Shlyapnikov, T. W. Hänsch, and I. Bloch, Nature (London) 429, 277 (2004); T. Kinoshita, T. Wenger, and D. S. Weiss, Science 305, 1125 (2004).

${ }^{14}$ G. Orso, Phys. Rev. Lett. 98, 070402 (2007).

${ }^{15}$ H. Hu, X.-J. Liu, and P. D. Drummond, Phys. Rev. Lett. 98, 070403 (2007).

${ }^{16}$ X. W. Guan, M. T. Batchelor, C. Lee, and M. Bortz, Phys. Rev. B 76, 085120 (2007).

${ }^{17}$ T. B. Bahder and F. Woynarovich, Phys. Rev. B 33, 2114 (1986).

${ }^{18}$ G. Roux, E. Orignac, P. Pujol, and D. Poilblanc, Phys. Rev. B 75, 245119 (2007).

${ }^{19}$ A. E. Feiguin and F. Heidrich-Meisner, Phys. Rev. B 76, 220508(R) (2007).

${ }^{20}$ M. Tezuka and M. Ueda, Phys. Rev. Lett. 100, 110403 (2008).

${ }^{21}$ G. G. Batrouni, M. H. Huntley, V. G. Rousseau, and R. T. Scalettar, Phys. Rev. Lett. 100, 116405 (2008).

${ }^{22}$ X.-J. Liu, H. Hu, and P. D. Drummond, Phys. Rev. A 76, 043605 (2007).

${ }^{23}$ F. Marsiglio, Phys. Rev. B 55, 575 (1997).

${ }^{24}$ G. I. Japaridze and A. A. Nersesyan, JETP Lett. 27, 334 (1978); Phys. Lett. 85A, 23 (1981); J. Low Temp. Phys. 47, 91 (1983); V. L. Pokrovsky and A. L. Talapov, Phys. Rev. Lett. 42, 65 (1979).

${ }^{25}$ H. Frahm and T. Vekua, J. Stat. Mech.: Theory Exp. 2008, P01007.

${ }^{26}$ F. Woynarovich, Phys. Rev. B 43, 11448 (1991); K. Penc and F. Woynarovich, Z. Phys. B: Condens. Matter 85, 269 (1991).

${ }^{27}$ F. D. M. Haldane, Proceedings of the International School of Physics "Enrico Fermi," Course CXXI "Perspectives in ManyParticle Physics," edited by R. A. Broglia and J. R. Schrieffer (North-Holland, Amsterdam, 1994), p. 5.

${ }^{28}$ M. Yamanaka, M. Oshikawa, and I. Affleck, Phys. Rev. Lett. 79, 1110 (1997); M. Oshikawa, M. Yamanaka, and I. Affleck, ibid. 78, 1984 (1997); P. Gagliardini, S. Haas, and T. M. Rice, Phys. Rev. B 58, 9603 (1998).

${ }^{29}$ D. Jaksch, C. Bruder, J. I. Cirac, C. W. Gardiner, and P. Zoller, Phys. Rev. Lett. 81, 3108 (1998)

${ }^{30}$ U. Schollwöck, Rev. Mod. Phys. 77, 259 (2005); S. R. White, Phys. Rev. Lett. 69, 2863 (1992).

${ }^{31}$ C.-S. Chuu, F. Schreck, T. P. Meyrath, J. L. Hanssen, G. N. Price, and M. G. Raizen, Phys. Rev. Lett. 95, 260403 (2005); T. P. Meyrath, F. Schreck, J. L. Hanssen, C.-S. Chuu, and M. G.
Raizen, Phys. Rev. A 71, 041604(R) (2005).

${ }^{32}$ T. Giamarchi, Quantum Physics in One Dimension (Clarendon, Oxford, 2004).

${ }^{33}$ Gao Xianlong, M. Rizzi, M. Polini, R. Fazio, M. P. Tosi, V. L. Campo, Jr., and K. Capelle, Phys. Rev. Lett. 98, 030404 (2007).

${ }^{34}$ F. Karim Pour, M. Rigol, S. Wessel, and A. Muramatsu, Phys. Rev. B 75, 161104(R) (2007).

${ }^{35} \mathcal{C}^{(0)}\left(k_{m}\right)$ is calculated from the noninteracting real-space correlator $\quad \mathcal{C}_{\ell \ell^{\prime}}^{(0)}=\left\langle\hat{c}_{\ell \uparrow}^{\dagger} \hat{c}_{\ell^{\prime} \uparrow}\right\rangle\left\langle\hat{c}_{\ell \downarrow}^{\dagger} \hat{c}_{\ell^{\prime} \downarrow}\right\rangle, \quad$ with $\left\langle\hat{c}_{\ell \sigma}^{\dagger} \hat{c}_{\ell^{\prime} \sigma}\right\rangle$ $=\sum_{n=1}^{N_{\sigma}} \varphi_{k_{n}}(\ell) \varphi_{k_{n}}\left(\ell^{\prime}\right)$.

${ }^{36}$ G. F. Giuliani and G. Vignale, Quantum Theory of the Electron Liquid (Cambridge University Press, Cambridge, 2005).

${ }^{37}$ L.-M. Duan, Phys. Rev. Lett. 96, 103201 (2006).

${ }^{38}$ I. Carusotto and Y. Castin, Phys. Rev. Lett. 94, 223202 (2005).

${ }^{39}$ E. Altman, E. Demler, and M. D. Lukin, Phys. Rev. A 70, 013603 (2004)

${ }^{40}$ M. Greiner, C. A. Regal, J. T. Stewart, and D. S. Jin, Phys. Rev. Lett. 94, 110401 (2005).

${ }^{41}$ L. Mathey, E. Altman, and A. Vishwanath, arXiv:cond-mat/ 0507108v3 (unpublished).

${ }^{42}$ A. Lüscher, A. M. Läuchli, and R. M. Noack, Phys. Rev. A 76, 043614 (2007).

${ }^{43}$ A. Lüscher, R. M. Noack, and A. M. Läuchli, arXiv:0712.1808v2 (unpublished).

${ }^{44}$ F. Schreck, L. Khaykovich, K. L. Corwin, G. Ferrari, T. Bourdel, J. Cubizolles, and C. Salomon, Phys. Rev. Lett. 87, 080403 (2001); A. Görlitz, J. M. Vogels, A. E. Leanhardt, C. Raman, T. L. Gustavson, J. R. Abo-Shaeer, A. P. Chikkatur, S. Gupta, S. Inouye, T. Rosenband, and W. Ketterle, ibid. 87, 130402 (2001); M. Greiner, I. Bloch, O. Mandel, T. W. Hänsch, and T. Esslinger, ibid. 87, 160405 (2001); H. Moritz, T. Stöferle, M. Köhl, and T. Esslinger, ibid. 91, 250402 (2003); T. Stöferle, H. Moritz, C. Schori, M. Köhl, and T. Esslinger, ibid. 92, 130403 (2004); H. Moritz, T. Stöferle, K. Guenter, M. Köhl, and T. Esslinger, ibid. 94, 210401 (2005).

${ }^{45}$ M. M. Parish, S. K. Baur, E. J. Mueller, and D. A. Huse, Phys. Rev. Lett. 99, 250403 (2007).

${ }^{46}$ A. Recati, P. O. Fedichev, W. Zwerger, and P. Zoller, Phys. Rev. Lett. 90, 020401 (2003).

${ }^{47}$ C. Kollath, U. Schollwöck, and W. Zwerger, Phys. Rev. Lett. 95, 176401 (2005)

${ }^{48}$ M. Polini and G. Vignale, Phys. Rev. Lett. 98, 266403 (2007). 